\title{
Korean Medicine for Treating Facial Palsy
}

\section{- A Literature Review of Case Reports -}

\author{
Hye In Jeong ${ }^{1+}$, Kyeong Han $\mathrm{Kim}^{2+}$, Yong Taek $\mathrm{Oh}^{3}$, Yoo Min $\mathrm{Choi}^{4}$, Beom Yong \\ Song ${ }^{4}$, Jong Uk Kim4, Tae-Han Yook ${ }^{4 *}$
}

\author{
${ }^{1}$ College of Korean medicine, Woosuk University, Jeonbuk, Korea \\ ${ }^{2}$ Department of Preventive medicine, College of Korean medicine, Woosuk University, Jeonbuk, Korea \\ ${ }^{3}$ Department of Diagnostics, College of Korean medicine, Woosuk University, Jeonbuk, Korea \\ ${ }^{4}$ Department of Acupuncture \& Moxibustion medicine, College of Korean medicine, Woosuk University, Jeonbuk, Korea
}

\section{Key Words}

facial palsy, case report, traditional Korean medicine

\section{Abstract}

Objectives: The aim of this study was to analyze case reports on and to study Korean medicine treatments of facial palsy.

Methods: Studies published after 2000 were selected by searching for the terms "facial palsy" and "Guanwasa" in four databases and case reports using Korean medicine moderating variables were extracted by two individual researchers.

Results: The House Brackmann (H-B) Scale (23 times, $65.7 \%)$, and Yanagihara Grading Scale (Y-system) (22 times, $62.8 \%$ ) were used to assess facial palsy. All case reports used acupuncture, while 19 case reports (51.3\%) used electro-acupuncture, and 8 case reports $(21.6 \%)$ used pharmacopuncture. Main meridian points used were ST6, ST4, GB14, TE23, LI20, BL2. Thirty-two case reports used herbal medicine (86.4\%) and both Ligigeo-

Received: Aug 09, 2018 Reviewed: Oct 23, 2018 Accepted: Nov 14, 2018

$@$ This is an Open-Access article distributed under the terms of the Creative Common Attribution Non-Commercial License (http://creativecommons.org/licenses/by-nc/4.0/) which permits unrestricted noncommercial use, distribution, and reproduction in any medium, provided the original work is properly cited.

(2) This paper meets the requirements of KS X ISO 9706, ISO 9706-1994 and ANSI/NISO Z39.48-1992 (Permanence of Paper). poong-san, Bojunglkgi-tang were used frequently.

Conclusion: To treat facial palsy, acupuncture was mainly used in conjunction with electro-acupuncture and pharmacopuncture. Most case reports also used herbal medicine.

\section{Introduction}

Facial palsy can be distinguished by two major types, central facial palsy and peripheral facial palsy. Most facial palsy occurs with the peripheral form [1]. Facial palsy mainly shows facial muscle paralysis to the affected side caused by facial nerve damage, along with pain in or behind the ear, altered sense of taste, sound hypersensitivity, tinnitus, drooling, and decreased tearing. The onset rate difference among age and sex shows clear distinction. Most facial palsy occurs on one side, and cases that affect both sides of the face occur rarely $(0.3 \%)$ [2]. In Korea, facial palsy occurs at the rate of 20 people out of 100,000 per year.

In Korean medicine, facial palsy is known as "Guanwasa" and is defined as the patient being unable to wrinkle the forehead on the affected side, unable to close the eyelid, with eye movement of the affected side moving upward and outward. Furthermore, ab-

*Corresponding Author

Tae-Han Yook. Department of Acupuncture \& Moxibustion Medicine, Woosuk University Hospital of Korean medicine, 46 Eoeun-ro, Wansan-gu, Jeonju, Jeonbuk 560-833, Korea

Tel: +82-63-220-8625 Fax: +82-63-220-8400

E-mail: nasiss@naver.com

+ Equally contributed.

(C) 2018 Korean Pharmacopuncture Institute

http://www.journal.ac 
normal tearing may occur, such as excessive or reduced tearing, drooping of the nosewing and nasojugal area which results in difficulty moving normally, altered sense of taste, decreased salivation, accompanied with pain in or behind the ear, headache, sound hypersensitivity and difficulty in hearing [3].

To diagnose facial palsy, various methods such as the House-Brackmann Grading System (H-B scale), Yanagihara Grading Scale (Y-system), and Weighted Regional Grading System (FEMA) are used. The H-B scale and Y-system are mainly used in the clinical field [4].

Treatment of facial palsy in conventional medicine includes corticosteroids or antivirus agents, and surgical treatments such as stellate ganglion block. When diagnosed with other diseases, internal treatment is initiated first, then followed by conservative treatment using steroid agents such as prednisone when no complications occur [5]. This type of treatment leads to shortening of the healing period, but the overall treatment results are still controversial [6]. The limits of conventional medicine treatment in facial palsy has led to increased interest in treatments with Korean medicine which focuses on conservative treatment.

Korean medicine therapeutic methods include acupuncture, herbal medicine, physical therapy, and others. Case reports show acupuncture treatment using filiform acupuncture needle treatments with electro-acupuncture or pharmacopuncture [7], or various treatments such as thread embedding acupuncture, Jung-an acupuncture [8], and Mi-so facial acupuncture [9]. Studies based on combined treatment using laser treatments such as Infrared (IR), Silver Spike Point (SSP) are continuously being published [10].

Numerous clinical studies on Korean medicine treatment of facial palsy are ongoing but literature review focusing on case reports to measure the current status of treatment in the clinical field is insufficient, especially overall studies on measuring tools, acupuncture, and herbal medicine. Therefore, this study aimed to generally investigate research trends, diagnostic methods, and treatments of facial palsy.

\section{Methods}

\subsection{Data sources}

The collected data were obtained using search engines including KISS (Korean Studies Information Service System), RISS (Research Information Service System), OASIS (Korean Medicine Information System), and NDSL (National Digital Science Library). Search words were "facial palsy" and "Guanwasa" and the retrieval date was July 2, 2018.

\subsection{Search strategy}

Studies of facial palsy using Korean medicine moderating variables were extracted by two individual researchers by reviewing the title and abstract of each study. The extract- ed studies were reviewed once again based on the original document to decide whether the research contents were suitable for this study. Case studies published after 2000 and before the retrieval date (June 2018) were included in this study.

The number of cases within one case report was not considered and were all included for study. Studies on complications associated with facial palsy such as pain in or behind the ear, otitis media, difficulty hearing, and strabismus were considered within the category of facial palsy and were included. Case studies in which Korean medicine treatment was not the main method or was not included were excluded.

\subsection{Data analysis}

Facial palsy studies using Korean medicine moderating variables were classified by the characteristic of moderating variables. Due to the lack of prior research, the classification criteria was based on internal discussions among researchers and was categorized mainly by use of acupuncture, herbal medicine, and other treatment (physical therapy, conventional medicine medication). Most studies used various therapeutic methods simultaneously, therefore the case reports were first classified, and the frequency of therapeutic methods were later observed.

Diagnostic and measuring tools documented in the study included tools used for evaluating facial palsy, and for measuring tools that are not related with facial palsy were additionally documented.

Case reports that clarified the affected side and non-affected side were all documented within the study, and case reports that did not clarify the affected side recorded the acupuncture points. If the number of acupuncture points included more than four points, the first four acupuncture points were documented. For conventional medicine medication, medicine taken only during the treatment period was documented. Past history which was recorded with the specific year was documented without particular marking, and past history without the specific year was documented with $\mathrm{Hx}$ (History). For cases that involved different therapeutic methods in one study, each therapeutic method was documented with marks such as \#1 and \#2.

Case reports that included more than two cases, or the evaluation tool, acupuncture points, and other therapeutic methods involved were identical, were analyzed as one case.

Acupuncture treatment studies clarified whether treatment was administered on the affected side, non-affected side, or both sides. Additional studies used acupuncture points without directional consideration. Therefore, when counting the acupuncture points, directions were not considered, and acupuncture treatment that was applied on both sides was considered as a one-time treatment instead of two treatments. Electro-acupuncture used two types of methods. One connected two different acupuncture points, and the other was applied electro-acupuncture on separate acupuncture points. The frequency rate was analyzed by each method. If acupuncture treatment was used but the name of the muscle was documented instead of the acupuncture point, the case report was excluded from ac- 
upuncture point analysis.

Case reports using herbal formulas consisted of cases that used a different formula on each case, or an identical formula for all cases. Therefore, if several cases in one paper used the same formula it was considered as one case. Additionally, a formula that was adjusted by adding or subtracting contents was considered as the original formula. For other therapeutic methods, the expression of treatment tools differed among the case reports and therefore was standardized and analyzed.

\subsection{Searching Result}

Based on the database results 1,087 articles published domestically were collected. After removing duplicated articles, 583 studies remained. Furthermore, 204 references were excluded, which were published before 2000 , leaving 379 articles to review for the abstract. Based on revision, 205 studies using conventional medicine moderating variables, 3 animal experimental studies, 5 trend analysis studies, and 118 clinical studies which are not case reports were excluded. As a result, a total of 37 references were selected for the analysis.

\section{Results}

\subsection{Summary of Case report}

Chronological analysis of the 37 case reports selected showed 10 case reports of facial palsy were published from 2000-2004, 8 published from 2009-2012, and 11 published from 2013-2017. The number of cases included in each case report differed and showed $23(62.1 \%)$ studies with one case, 7 studies (18.9\%) with two cases, and 7 studies (18.9\%) with more than three cases.

All studies included acupuncture treatment, 19 studies (51.3\%) used electro-acupuncture additionally, and 8 studies (21.6\%) used additional pharmacopuncture. Six studies (16.2\%) used acupuncture, electro-acupuncture, and pharmacopuncture simultaneously. Thirty-two case reports (86.4\%) used herbal medicine and 13 studies (35.1\%) used conventional medicine medication simultaneously. Eleven case reports $(29.7 \%)$ used conventional medicine medication and herbal medicine simultaneously. Thirty-five case reports (94.5\%) used measuring tools to evaluate patient improvement rate. (Table 1)

\subsection{Analysis of measuring tools}

Thirty-five case reports out of 37 used evaluation tools. The H-B Scale (23 times, 65.7\%) and Y-system (22 times, $62.8 \%$ ), and Digital infrared thermal imaging (DITI) (5 times, $14.2 \%$ ) were used in sequence. Numeral rating scale (NRS) was used in 3 studies (8.5\%), and visual analog scale (VAS) was used in 6 studies (17\%) to evaluate pain in or behind the ear or tinnitus.

\subsection{Treatment tools}

\subsubsection{Acupuncture}

Out of 37 case reports, acupuncture was used a total of 453 times. For acupuncture treatment, the name of acupuncture points and number of acupuncture points used for treatment is as follows:

ST6 (38 times, 8.3\%), ST4 (34 times, 7.5\%), TE23 (27 times,

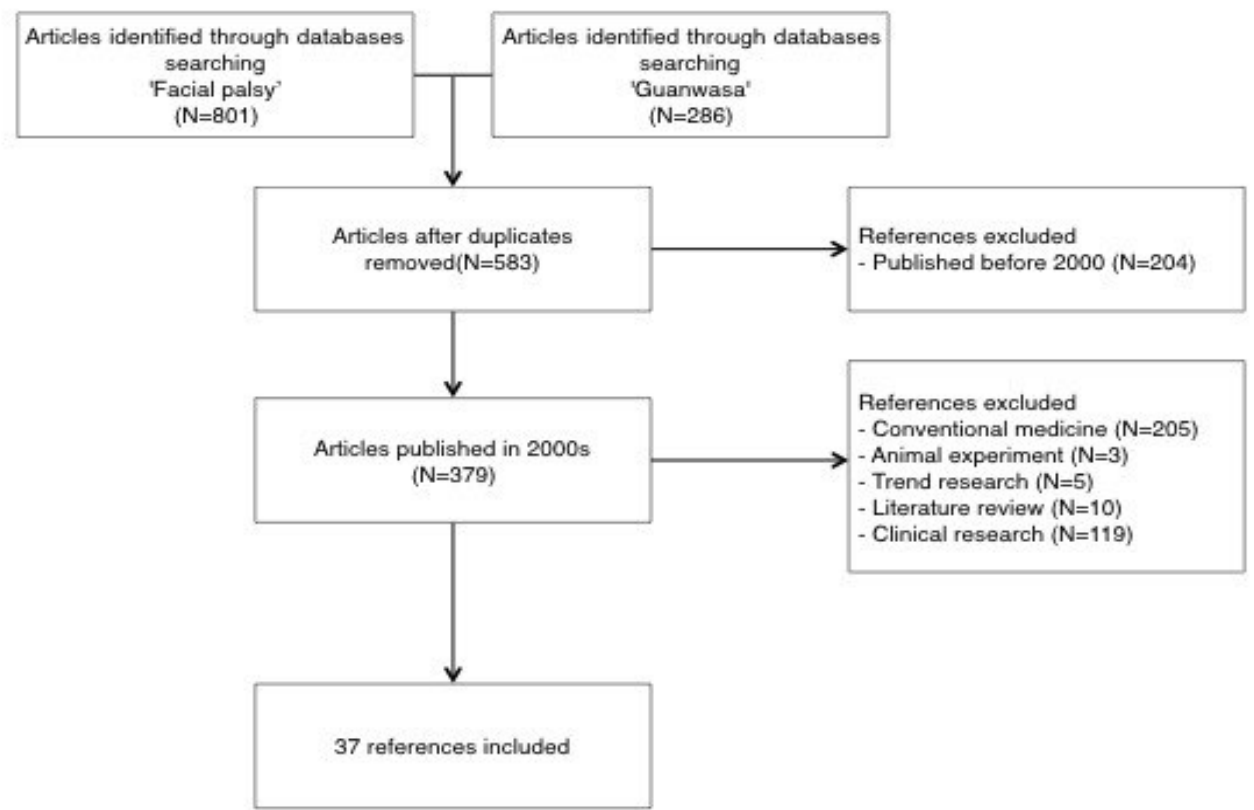

Figure 1 Flow chart of research 
5.9\%), GB14 (27 times, 5.9\%), GV26 (27 times, 5.9\%), LI4 (26 times, 5.7\%), LI20 (23 times, 5\%), BL2 (23 times, 5\%), ST2 (20 times, 4.4\%), SI18 (18 times, 3.9\%), CV24 (18 times, $3.9 \%$ ), TE17 (16 times, 3.5\%), ST36 (16 times, 3.5\%), ST7 (12 times, 2.6\%), EX-HN4 (10 times, 2.2\%), EX-HN5 (10 times, $2.2 \%$ ), ST1 (8 times, 1.7\%), LR3 (8 times, 1.7\%), EX-HN3 (7 times, $1.5 \%$ ), GB20 (7 times, $1.5 \%$ ).

Nineteen $(51.3 \%)$ case reports used electro-acupuncture 26 times. The use of ST4-ST6 was the most frequently used (13 times, 50\%), followed by TE23-BL2 (3 times, 11.5\%), ST7-SI18 (2 times, 7.6\%). In the method of using electro-acupuncture for each acupuncture point, ST6 was the most frequently used point (9 times, $11.2 \%$ ), followed by GB14, ST4 (8 times, 10\%), LI20, BL2 (7 times, 8.7\%), SI18 (6 times, 7.5\%), ST7, and EX-HN3 (4 times, 5\%).

Case reports that used pharmacopuncture totaled 8 (21.6\%), 12 times. Types of pharmacopuncture mainly used were Hominis Placenta, Hwangryunhaedok-tang, and bee venom acupuncture, each used 3 times (25\%).

\subsubsection{Herbal medicine}

Out of 37 case reports, case reports that used herbal medicine totaled 32 (86.4\%), 49 times. Frequently used herbal medicines were Ligigeopoong-san (15 times, 16.8\%), Bojunglkgi-tang (9 times, 10.1\%), and Gyeonjeong-san, Bogigeopung-san, Ligigyeonjeong-san (5 times, 5.6\%).

\subsubsection{Other treatment}

Twenty-nine case reports used other treatments, and out of 29 cases, IR was used most frequently (15 times, $51.7 \%$ ), followed by facial massage (13 times, $44.8 \%$ ) and SSP (Silver spike point) (13 times, $44.8 \%$ ). Facial massage, hot-pack (5 times, 17.2\%), moxibustion, carbon, and laser were also used ( 4 times, 13.7\%).

\section{Discussion}

This study collected and analyzed case reports of facial palsy using Korean medicine treatment. As a result, chronological analysis showed that continuous publication of case reports on facial palsy has been ongoing. Ten case reports of facial palsy were published from 2000-2004, 8 published from 2009-2012, and 11 published until 2017, indicating that Korean medicine treatment of facial palsy has been continuously used and related research has also been conducted.

Evaluation tools of facial palsy used in clinical practice should be able to accurately evaluate the level of paralysis in a short period of time, and also requires high reproductibility [4]. Electric diagnostic findings such as electromyogram (EMG) and electroneuronography (ENoG) are insufficient evaluating the level of facial function recovery, therefore grading systems to measure functional recovery levels are frequently used [12].

Grading systems used to evaluate facial palsy consist of two types; the general method that measures the overall condition of facial palsy and accompanied symptoms simultaneously, and the special method that first measures the paralysis condition by each area and then grades the overall disability level and accompanied symptoms. The $\mathrm{H}$-B scale is mainly used in the general method, while the Y-system is used in the special method [13]. Based on the analysis in this study, like preceding studies, the H-B scale (23 times, 65.7\%) and Y-system (22 times, 62.8\%) are used with high frequency. The H-B scale shows low deviation between measures and has simple evaluation methods which are easy to use and quickly evaluated. The Y-system seems to be frequently used in clinical practice since it enables objective and quantitative evaluation of facial function without a specific tool, and can sensitively measure a patient's change of condition [4]. Other evaluation tools such as Sunnybrook facial grading system (SFGS) or detailed evaluation of facial symmetry (DEFS) were used, but the frequency and reproducibility were very low and the measuring time is long compared to the $\mathrm{H}-\mathrm{B}$ scale and Y-system.

The most frequently used acupuncture points for acupuncture and electro-acupuncture treatment were ST6 followed by ST4, GB14, TE23, LI20, BL2, and GV26. In Korean medicine literature, it is recorded that ST4, ST6, and LI4 are used for Guanwasa [14] and it is thought that these acupuncture points are located in the meridians (stomach channel of foot Yangming, gallbladder channel of foot Shaoyang, bladder channel of foot Taiyang, large intestine channel of the hand Yangming) that passthrough the facial area. Among the frequently used acupuncture points, LI4, although not located in the facial area, especially the starting point of Stomach Channel of Foot Yangming, shows skin temperature changes in the facial area when acupuncture needles are placed there. this shows the correlation of LI4 and the facial area. Considering that Guanwasa is a disease caused by wind-cold in the facial nerve, controlling cold and heat by skin temperature can be related to the treatment of facial palsy [11]. Studies indicate that electro-acupuncture shows significant treatment effects on facial palsy [16]. Electro-acupuncture is also used frequently since it can be used at any part of the human body by applying intensive stimulation for chronic pain or paralysis diseases [17] and shows advantage in paralyzed nerves or myoatrophy [18].

Hominis Placenta, Hwangryunhaedok-tang, and bee venom acupuncture were mainly used for pharmacopuncture. Hominis Placenta pharmacopuncture is used for regenerating tissues, developing antibodies, enhancing resistance and supplementing lacking yoon in meridians [19]. Bee venom acupuncture can cause side effects such as anxiety, rashes, and vomiting when allergic reaction occurs, but is widely used in inflammation and paralysis diseases due to the analgesic effect, anti-inflammation and effectively removing wind-dampness [20]. Hwangryunhaedok-tang pharmacopuncture has wind factor scattering effect and anti-inflammation effects [21]. The facial nerve has a long pathway compared to other cranial nerves. It passes through a narrow pathway which leads to the facial nerve being easily damaged during surgery of the middle ear or temporal bone, injury or infection, which is why anti-inflammation pharmacopuncture is used frequently [22].

Ligigeopoong-san, the most frequently used formula, is 
Table 1 Traditional Korean medicine therapeutic methods of facial palsy

\begin{tabular}{|c|c|}
\hline Classification & Acupoint/ Herbal medicine/Treatment (number of case,\%) \\
\hline \multirow{5}{*}{ Acupuncture } & $\begin{array}{l}\text { ST6(38, 8.3\%) ST4(34, 7.5\%) TE23, GB14(27, 5.9\%) LI4(26, } \\
5.7 \%) \text {, LI20, BL2(23, 5\%) }\end{array}$ \\
\hline & ST4-ST6(13, 50\%) TE23-BL2 $(3,11.5 \%)$ \\
\hline & ST7-SI18(2, 7.6\%) \\
\hline & ST6(9, 11.2\%) GB14(8, 10\%), ST4(8, 10\%) LI20, BL2(7, 8.7\%) \\
\hline & $\operatorname{SI} 18(6,7.5 \%)$ \\
\hline Pharmacopuncture & $\begin{array}{l}\text { Hominis Placenta }(3,25 \%), \text { Hwangryunhaedoktang }(3,25 \%) \text {, } \\
\text { Bee venom }(3,25 \%)\end{array}$ \\
\hline Herbal medicine & $\begin{array}{l}\text { Ligigeopoong-san }(15,16.8 \%), \quad \text { Bojunglkgi-tang }(9,10.1 \%) \text {, } \\
\text { Gyeonjeong-san }(5,5.6 \%), \quad \text { Bogigeopung-san }(5,5.6 \%) \text {, } \\
\text { Ligigyeonjeong-san }(5,5.6 \%)\end{array}$ \\
\hline Other treatment & $\begin{array}{l}\operatorname{IR}(15,51.7 \%) \text { massage }(13,44.8 \%) \operatorname{SSP}(11,37.9 \%) \text {, Facial } \\
\text { exercises , Hot pack }(5,17.2 \%) \text { moxa, Carbon, Laser }(4,13.7 \%)\end{array}$ \\
\hline
\end{tabular}

Table 2 List of case report of treatment Korean medicine of facial palsy

\begin{tabular}{|c|c|c|c|c|}
\hline classification & subjects(onset) & tool & Korean medicine treatments & other treatment \\
\hline Jang (2016) & F/19 Lt. facial palsy (2015.03) & $\begin{array}{l}\text { H-B scale } \\
\text { Y-system } \\
\text { DITI } \\
\text { NRS } \\
\text { (difficulty hearing, tinnitus) }\end{array}$ & $\begin{array}{l}-0.25 \times 30 \mathrm{~mm} 1 \text { time/day, } 15 \text { mins } \\
\text { - non-affected side: LI4 LU9 SP3 LI1 } 1 \text { etc } \\
\text { - affected-side: LI20 ST2 ST3 ST4 etc } \\
\text { - } 0.25 \text { cc x } 18 \text { times pharmacopuncture } \\
\text { - Jungsongouhyu GB20 TE17 }\end{array}$ & $\begin{array}{l}\text { - Depakote ER Tab } 250 \text { mg 1T\#1 (1-0-0) } \\
\text { Depakote ER Tab } 500 \mathrm{mg} \text { 1T\#1 (1-0-0) } \\
\text { Gliatilin Soft Cap 3T\#3 (1-1-1) } \\
\text { Nexium Tab } 20 \text { mg 1T\#1 (1-0-0) } \\
\text { Paramacet Tab 3T\#3 (1-1-1) } \\
\text { - IR, SSP, Laser } 1 \text { time/day }\end{array}$ \\
\hline Son (2011) & $\begin{array}{l}\text { \#1 F/15 Rt. facial palsy (2010.4) } \\
\text { \#2 F/39 Lt. facial palsy (2011.3) } \\
\text { Hx tuberculosis }\end{array}$ & H-B scale & $\begin{array}{l}\# 1 \\
-0.25 \times 40 \mathrm{~mm} 1 \text { times/day, } 20 \text { mins, direct acupuncture } \\
\text {-2010.11.12.-2011.3.25. acupuncture treatment } 2 \text { times/week } \\
\text { - both sides: ST36, LI4, LR3 } \\
\text { - affected-area: TE17, ST4, ST6, SI18 etc } \\
\text { - Soyangin Dokhwaljihwang-tang Gagam } 3 \text { times /day } 120 \mathrm{cc} \\
\# 2 \\
\text { - 0.25x40mm } 1 \text { time/day direct acupuncture } \\
\text { - 2011.6.29.-2011.9.23. acupuncture treatment } 3 \text { times/week } \\
\text { - acupuncture area same as \#1 } \\
\text { - Soyangin Dokhwaljihwang-tang Gagam } 3 \text { times/day } 120 \mathrm{cc}\end{array}$ & $\begin{array}{l}\text { - IR on affected-side } \\
\text { - Carbon, SSP, facial massage }\end{array}$ \\
\hline Lee (2017) & $\begin{array}{l}\text { F/77 Rt. Facial palsy (2016.11) } \\
50 \text { Rt. earache, pus in middle ear } \\
\text { '16 Rt. Tympanic membrane } \\
\text { perforation surgery } \\
15 \text { hypertension medication }\end{array}$ & $\begin{array}{l}\text { H-B scale } \\
\text { Y-system } \\
\text { VAS(otitis media) }\end{array}$ & $\begin{array}{l}\text { - } 0.18 \times 40 \mathrm{~mm} 2 \text { times/day, 15-20mins, (1 time on Sundays), direct } \\
\text { acupuncture } \\
\text { - 2016.11.17.-12.27 } \\
\text { - both sides: EX-HN3 EX-HN4 TE23 GB1 etc } \\
\text { - pharmacopuncture: Hwangryunhaedok-tang, SI19 TE17 } \\
\text { - electro-acupuncture: ST4-ST6, EX-HN4 -TE23 } \\
\text { - herbal medicine: } 3 \text { packs/day 120cc } \\
\text { - 11.17-23: GamiLigigyeonjeong-san } \\
\text { - 11.24-12.7: SamulanshintangGami } \\
\text { - 12.8-27: HyunggyeyungyotanGami }\end{array}$ & $\begin{array}{l}\text { - Thread embedding acupuncture } 3.0 \mathrm{~cm} \text {, } \\
4.0 \mathrm{~cm} 5-7 \text { each } \\
\text { - GB14 ST6 ST4 ST3 SI18 } \\
\text { - antihypertensive } / 1-0-0 \\
\text { - } 11.17-20 \\
\text { - Adrenal hormones } / 7-0-2 \\
\text { - Antipyretic } / 1-0-1 \\
\text { - peptic ulcer medication } / 1-0-1 \\
\text { - } 11.21-25 \\
\text { - Adrenal hormones } / 4-0-2 \\
\text { - Antipyretic } / 1-0-1\end{array}$ \\
\hline
\end{tabular}




\begin{tabular}{|c|c|c|c|c|}
\hline classification & subjects(onset) & tool & Korean medicine treatments & other treatment \\
\hline & & & & $\begin{array}{l}\text { - Antacid } / 1-0-1 \\
-11.26-30 \\
\text { - Adrenal hormones } / 4-0-0 \\
\text { - antacid / 1-0-0 } \\
\text { - physical therapy including electrical } \\
\text { treatment }\end{array}$ \\
\hline Kang (2016) & $\begin{array}{l}\text { F/52 Facial palsy both sides, otitis } \\
\text { media (2015.2) } \\
\text { '86 Frequent relapse after diagnosis. } \\
\text { ENT oral medication } \\
\text { '00 Diagnosed D.M - on oral } \\
\text { medication and hypodermic injection } \\
\text { '13 Lt. back herpes zoster - full } \\
\text { recovery after oral medication } \\
\text { '15 } 5 \text { dental implants, } 3 \text { dental } \\
\text { crowns } \\
\text { '15. } 10 \text { Rt. Ear sudden deafness - full } \\
\text { recovery after oral medication } \\
\text { '16. } 1 \text { hyperthyroidism - on oral } \\
\text { medication }\end{array}$ & $\begin{array}{l}\text { H-B scale } \\
\text { Y-system } \\
\text { Paralysis level of muscle } \\
\text { (criterion- }++++ \text { ) }\end{array}$ & $\begin{array}{l}\text { - } 0.20 \times 30 \mathrm{~mm} 2 \text { times/day, } 20 \text { mins (inpatient) / } 1 \text { time/day, } 20 \text { mins } \\
\text { (outpatient) } \\
\text { - both sides: ST4 ST6 ST3 GV26 etc } \\
\text { - Pharmacopuncture: Hominis placenta (Mon, Wed, Fri) } \\
\text { Hwangryunhaedok-tang (Tues, Thur, outpatient) } \\
\text { - TE23 EX-HN3 SI19 ST4 ST6 etc } \\
\text { - electro-acupuncture: ST4-ST6, TE23-BL2 } \\
\text { - herbal medicine } \\
\text { - 02.25 3 times/day 120cc CheunggansoyosanGami } \\
\text { - } 04.11 \sim \text { paranasal sinusitis-2, pus in ear, diagnosis of otitis media }\end{array}$ & $\begin{array}{l}\text { - moxibustion (Dongbang mugwort-charcoal, } \\
\text { Haetnim Shingigu) TE17 } \\
\text { - } 2 \text { times/day, } 15 \text { mins (inpatient) / } 1 \text { time/day, } \\
20 \text { mins (outpatient) } \\
\text { - IR on facial area } \\
\text { - } 2 \text { times/day (inpatient) } / 1 \text { time/day, } 20 \text { mins } \\
\text { (outpatient) } \\
\text { - Cupping } \\
\text { - Wet cupping } 21 \text { times TE17 GB20 } \\
\text { - Dry cupping } 54 \text { times GB20 GB21 } \\
\text { - PT EST, Laser, SSP (started from recovery } \\
\text { period) } 1 \text { time/day } \\
\text { - EST, SSP } 1 \text { time/day (outpatient) } \\
\text { - Otitis media treatment Yigang Laser - } 2 \\
\text { times/day x } 7 \text { days, } 1 \text { time/day (outpatient) }\end{array}$ \\
\hline Kim (2003) & $\begin{array}{l}\text { F/54 Lt. facial palsy (2003.6) } \\
02 \text { Diagnosed Hypertension, D.M - } \\
\text { medication } \\
03 \text { Diagnosed periarthritis began } \\
\text { medication - after improvement of } \\
\text { symptoms stopped medication }\end{array}$ & $\begin{array}{l}\text { Y-system } \\
\text { VAS(facial pain, pain in } \\
\text { and behind the ear, } \\
\text { headache) }\end{array}$ & $\begin{array}{l}\text { - } 0.30 x 40 \mathrm{~mm} \\
\text { - non-affected side: LI4 PC6 etc } \\
\text { - affected-side: ST4 ST6 PC7 etc } \\
\text { - electro-acupuncture: ST7-SI18, ST4-ST6, BL2-TE23 } \\
\text { - pharmacopuncture: bee venom acupuncture, facial area of affected- } \\
\text { side GB14, ST2, SI18, ST4 etc } \\
\text { - hand acupuncture: TE17, ST6, ST7, EX-HN5 } \\
\text { - herbal medicine } \\
\text { - early stage pain in and behind the ear severe - Wugakseongma-tang, } \\
\text { after reduced Ligigeopoong-san } \\
\text { - after acute stage Ligigyeonjeong-san, GamiSsangbo-tang medication }\end{array}$ & $\begin{array}{l}\text { - antivirus agents, corticosteroids combined } \\
\text { treatment }-1 \text { week after hospitalization } \\
\text { - carbon laser on facial area during } \\
\text { acupuncture treatment } \\
\text { - SSP FES } 1 \text { time/day }\end{array}$ \\
\hline $\begin{array}{l}\text { Lee } \\
(2013)\end{array}$ & $\begin{array}{l}\text { \#1 F/9 Rt. Facial palsy (2012.11) } \\
\text { 11 Conventional medicine } \\
\text { medication after diagnosed of gastritis } \\
\text { \#2 M/10 Rt. Facial palsy (2012.12) } \\
\text { \#3 F/21m Rt. Facial palsy (2012.12) } \\
\text { \#4 M/9 Rt. Facial palsy (2013.1) } \\
\text { Hx ankle sprain } \\
\text { \# M/7 Rt. Facial palsy (2013.1) } \\
\text { 11.6 diagnosed of otitis media, } \\
\text { improved after surgery. Rt. Facial } \\
\text { nerve palsy (Guanwasa) }\end{array}$ & $\begin{array}{l}\text { H-B scale } \\
\text { ENoG }\end{array}$ & $\begin{array}{l}\text { - } 0.20 \times 30 \mathrm{~mm} 2 \text { times/day, } 15 \mathrm{mins} \\
\text { - Removed acupuncture needles when difficult to leave needles in } \\
\text { during treatment } \\
\text { - acupuncture points: GV20 EX-HN3 EX-HN5 TE23 ST1 etc } \\
\text { - electro-acupuncture: ST4-ST6, stimulated } 15 \text { mins } \\
\text { - herbal medicine } \\
\text { - \#1 hyangsapyungwisan - Banhabaekchulchunma-Tang + Gyeonjeong- } \\
\text { san } \\
\text { - Hyangsayukgunja-tang } \\
\text { - \#2 Banhabaekchulchunma-Tang + Gyeonjeong-san } \\
\text { - \#3 Samsoeum + Gyeonjeong-san - Bojunglkgi-tang + Gyeonjeong-san } \\
\text { - \#4 Ligigeopoong-san - Soongiwhalhyultang } \\
\text { - \#5 Banhabaekchulchunma-Tang + Gyeonjeong-san - Jesubsungitang }\end{array}$ & $\begin{array}{l}\text { - IR on affected area } \\
\text { - Laser on acupuncture points } 2 \text { times/day, } 1 \\
\text { sec/acupuncture point and time } \\
\text { - low frequency electro-acupuncture }\end{array}$ \\
\hline Choo (2009) & $\begin{array}{l}\text { \#1 M/51 Rt. Facial palsy (2008.5) } \\
\text { '06 HT(+) Cognition W-med Od Po } \\
\text { \#2 F/70 Rt. Facial palsy (2008.6) } \\
\text { Hx HT(+) DM(+) W-med Qd Po }\end{array}$ & $\begin{array}{l}\text { H-B scale } \\
\text { DITI }\end{array}$ & $\begin{array}{l}-0.20 \times 40 \mathrm{~mm} 1 \text { time/day, } 20 \text { mins } \\
\text { - Facial acupuncture (frontal belly, orbicularis oculi muscle, mimic } \\
\text { muscles, levator labii superioris etc) } \\
\text { - herbal medicine } \\
\text { - GamiLigigyeonjeong-san, Bogigeopung-san }\end{array}$ & - SSP, Laser 1 time/day, 6 times/week \\
\hline Oh (2009) & $\begin{array}{l}\text { \#1 F/49 Lt. facial palsy (2008.10) } \\
\text { ' } 93 \mathrm{Lt} \text { arm Fx. surgery } \\
\text { \#2 M/62 Lt. facial palsy (2009.3) } \\
\text { '70 Gastric ulcer 1-2 years medication } \\
\text { '05 HIVD of L-spine \& Both knee } \\
\text { joint O.A. } \\
\text { '07 Brain MRI 'W.N.L' }\end{array}$ & $\begin{array}{l}\text { H-B scale } \\
\text { Y-system } \\
\text { VAS(pain in and behind } \\
\text { the ear) }\end{array}$ & $\begin{array}{l}\text { - } 0.25 \times 30 \mathrm{~mm}, 0.18 \mathrm{x} 8 \mathrm{~mm} \text { (Hand needle) } 2 \text { times/day, } 15 \text { mins } \\
\text { - non-affected side: LI4 SI3 ST36 ST41 etc } \\
\text { - affected-side: EX-HN3 BL2 EX-HN4 TE23 etc } \\
\text { - electro-acupuncture: } 30 \mathrm{~Hz} \text {, same as above } \\
\text { - herbal medicine: GamiBoikyangwie-tang } \\
\text { - pharmacopuncture: bee venom acupuncture, pain in and behind the } \\
\text { ear treatment }\end{array}$ & $\begin{array}{l}\bullet \text { indirect moxibustion } \\
\text { (Dongbanggu Poongnyun } \\
\text { mugwort moxibustion) } \\
\text { - } 3 \text { times/day ST4, ST6, LI20, SI18, TE23, } \\
\text { BL2 }\end{array}$ \\
\hline Lee (2015) & $\begin{array}{l}\text { F/61 Sequela of facial palsy (paralysis } \\
\text { of lt. area and lip and mouth area, } \\
\text { contraction of lt. area and mouth } \\
\text { area. Tearing and convulstion) } \\
\text { Hx lt. facial palsy several years ago, } \\
\text { hospitalized in conventional medical } \\
\text { hospital, treatment in Korean }\end{array}$ & H-B scale & $\begin{array}{l}\text { - } 0.20 \times 30 \mathrm{~mm} \text { Jung-an acupuncture } 1 \text { time/day } \mathrm{x} 8 \text { days } \\
\text { - direct acupuncture on both sides of sternocleidomastoid muscle and } \\
\text { instant remove of needle } \\
\text { - crosswayed acupuncture on digastric muscle, lateral pterygoid and } \\
\text { instant remove of needle } \\
\text { - direct or crosswayed acupuncture on auricularis, temporalis, masseter } \\
\text { muscle, levator labii superioris, orbicularis oculi muscle, mimic }\end{array}$ & - facial massage \\
\hline
\end{tabular}




\begin{tabular}{|c|c|c|c|c|}
\hline classification & subjects(onset) & tool & Korean medicine treatments & other treatment \\
\hline
\end{tabular}

medicine clinic occasionally

muscle following the grain and direction of muscle, 20 mins

\begin{tabular}{|c|c|c|c|c|}
\hline $\begin{array}{l}\text { Ko } \\
(2004)\end{array}$ & $\begin{array}{l}\text { F/56 Vertigo, Diplopia, Lt. facial } \\
\text { palsy } \\
\text { Hx Hypertension, D.M. cognition ( } 10 \\
\text { years) p.o. med }\end{array}$ & $\mathrm{X}$ & $\begin{array}{l}\text { - } 1 \text { time/day } \\
\text { - acupuncture points: BL1 GB1 ST2 GB14 etc } \\
\text { - Seven Acupoints for Stroke Objectives ST6 ST4 } \\
\text { - herbal medicine } \\
\text { - Sunghyangchungisan - diagnosed fatigue - Bojunglkgi-tang }\end{array}$ & $\begin{array}{l}\text { - cerebral infarction } \\
\text { - Thrombolytic agent, antiplatelet agent }\end{array}$ \\
\hline Cho (2010) & $\begin{array}{l}\text { \#1 F/18 Lt. facial palsy (2010.4) } \\
\text { 04 } 2 \text { months treatment for Peripheral } \\
\text { facial palsy - full recovery } \\
\text { \#2 F/47 Lt. facial palsy (2010.3) } \\
\text { 00 Medication after diagnosed } \\
\text { hypertension } \\
\text { \#3 M/31 Lt. facial palsy (2010.4) } \\
10 \text { surgical procedure after diagnosed } \\
\text { of brain tumor - on medication } \\
\text { \#4 M/52 Lt. facial palsy (2010.5) } \\
08 \text { medication after diagnosed of } \\
\text { hypertension } \\
80 \text { medication after diagnosed of } \\
\text { acute nephritis } \\
\text { \#5 F/54 Rt. facial palsy (2010.5) } \\
\text { \#6 F/68 Rt. facial palsy (2005.5) } \\
95 \text { medication after diagnosed of } \\
\text { herniation of lumbar disk } \\
09 \text { medication after diagnosed of } \\
\text { hyperlipidemia }\end{array}$ & $\begin{array}{l}\text { H-B scale } \\
\text { Y-system }\end{array}$ & $\begin{array}{l}-0.20 \times 30 \mathrm{~mm} 2 \text { times/week } \times 3 \text { weeks, } 15 \text { mins } \\
\text { - direct or sidewayed acupuncture following orbicularis oculi muscle, } 4 \\
\text { above, } 4 \text { below middle of the eyeball } \\
\text { - affected-side: GV26 CV24 TE17 ST4 ST6 etc Perpendicular needling } \\
\text { or ST4-ST6 Tu-ja } \\
\text { - herbal medicine: Bojunglkgi-tang, Yukmizihwangtang, Soyosan }\end{array}$ & - manual therapy, ICT \\
\hline $\operatorname{Kim}(2001)$ & $\begin{array}{l}\text { \#1 F/61 Lt. facial palsy (2001.4) } \\
\text { ' } 97 \text { cerebral infarction } \\
\text { ' } 94 \text { hypertension cognition } \\
\text { \#2 F/62 Lt. facial palsy (2001.4) } \\
\text { '99 hypertension } \\
\text { \#3 F/32 Lt. facial palsy (2001.6) } \\
\text { 99 L-spine HNP cognition } \\
\text { \#4 F/51 Lt. facial palsy (2001.6) } \\
\text { \#5 M/52 Rt. facial palsy (2001.7) } \\
\text { '96 Hypertension cognition }\end{array}$ & $\begin{array}{l}\text { Measuring area of facial } \\
\text { palsy }(+/ \text { ) } \\
\text { Lucille Daniels method } \\
\text { (survey) }\end{array}$ & $\begin{array}{l}\text { - filiform acupuncture: GB14 ST4 ST6 ST2 LI4 EX-HN5 GV26 GB34 etc } \\
\text { - electro-acupuncture: ST6-ST4, GB34 } \\
\text { - pharmacopuncture: Hominis Placenta, GB14 ST4 ST6 ST2 EX-HN5 etc } \\
\text { 0.1 1cc/ Hwangryunhaedoktang, GB21 } \\
\text { - herbal medicine } \\
\text { - \#1 joripewontang, Ligigeopoong-san, } \\
\text { Gajengyok-tang(SamultangGagam) } \\
\text { - \#2 1st Chengsangdoktong-tang, 2nd Yeoldahansotang } \\
\text { - \#3 Gamisayuktang } \\
\text { - \#4 Chengsangdoktong-tang, Ligigeopoong-san, chungsimyeunjatang } \\
\text { - \#5 Ligigeopoong-san, Hyungbangjihwang-tang }\end{array}$ & \\
\hline Cho (2000) & $\begin{array}{l}\text { F/55 Rt. facial palsy ( } 2 \text { years ago), } \\
\text { visit due to strabismus (2000.4) } \\
\text { Hx. urinary incontinence surgery ( } 4 \\
\text { years ago) } \\
\text { both side knee arthritis ( } 3-4 \text { years ago) } \\
\text { sinusitis surgery (age } 17 \text { ) }\end{array}$ & $\begin{array}{l}\text { Measuring area of facial } \\
\text { palsy }(+/-) \\
\text { Lucille Daniels method } \\
\text { (survey) } \\
\text { MET }\end{array}$ & $\begin{array}{l}\text { - filiform acupuncture: GV20 TE17 ST6 ST4 etc } \\
\text { - electro-acupuncture: ST6-ST4, ST6-ST5, ST3-ST1, GV26-SI18 etc } \\
\text { - herbal medicine: GamiBoik-tang, Oyaksoonkisan, Jamyangturak- } \\
\text { tang }\end{array}$ & $\begin{array}{l}\text { - medication } \\
\text { - 6/13 Gliclazide } 80 \mathrm{mg} \# 1 \\
\text { - 6/18 Gliclazide } 80 \mathrm{mg} \# 1 \text { Voglibose } 0.6 \mathrm{mg} \# 2 \\
\text { - } 6 / 29 \text { Gliclazide } 80 \mathrm{mg} \# 1 \\
\text { Voglibose } 0.6 \mathrm{mg} \text { Metformin } \mathrm{HCl} 1000 \mathrm{mg} \# 2 \\
\text { - 7/18 Gliclazide } 80 \mathrm{mg} \# 1 \\
\text { Voglibose } 0.6 \mathrm{mg} \text { Metformin } \mathrm{HCl} 1000 \mathrm{mg} \# 2\end{array}$ \\
\hline Ha (2007) & $\begin{array}{l}\mathrm{M} / 11 \text { simultaneous facial palsy on } \\
\text { both sides (2006.9.23-Lt., 28-Rt.) } \\
\text { Hx } 12 \text { month hospitalized treatment of } \\
\text { asthma, on conventional medicine } \\
\text { medication } \\
\text { Surgery for pierced wound at outer } \\
\text { area of eye (age 4) }\end{array}$ & $\begin{array}{l}\text { H-B scale } \\
\text { Y-system }\end{array}$ & $\begin{array}{l}\bullet \text { both sides } 1 \text { time/day, } 30 \text { mins - acupuncture points: ST4 ST6 GV26 } \\
\text { ST2 } \\
\text { - herbal medicine - } 2 \text { chubs for } 3 \text { separate doses } \\
\text { - 9/29 10/14 Cheongungkyeoji-tang } \\
-10 / 15 \sim 10 / 27 \text { Palmulgunja-tang }\end{array}$ & \\
\hline $\operatorname{Kim}(2002)$ & F/32 Rt. Facial palsy (2000.8) & H-B scale & $\begin{array}{l}\text { - } 0.25 \times 30 \mathrm{~mm} \text { acupuncture treatment on both sides simultaneously, } 20 \\
\text { mins } \\
\text { - acupuncture points: ST2, GB14, ST8, BL2 etc, Stomach-tonification } \\
\text { in turn } \\
\text { - electro-acupuncture: same as above } \\
\text { - herbal medicine: GamiLigigeopoong-san }\end{array}$ & $\begin{array}{l}\text { - IR during acupuncture treatment } \\
\text { - corticosteroids } 3 \text { times/day } \\
\text { (first week of visit) } \\
\text { - facial massage, frequent exercise } \\
\text { of expression muscles }\end{array}$ \\
\hline Jang (2013) & $\begin{array}{l}\mathrm{M} / 15 \quad \text { Facial palsy both sides } \\
(2011.12)\end{array}$ & H-B scale & $\begin{array}{l}\text { - } 0.25 \times 40 \mathrm{~mm} \text { (proximal area) } 0.30 \times 15 \mathrm{~mm} \text { (distal area) } 1 \text { time/day, } 30 \\
\text { mins } \\
\text { - acupuncture points: ST6 ST4 GV26 CV24 etc } \\
\text { - electro-acupuncture: ST6-ST4 ST7-SI18 EX-HN4 (HN4)-GB14 } \\
\text { - herbal medicine: OyaksoonkisanGami. } 20 \text { chub } 100 \mathrm{cc} 2 \text { times/day }\end{array}$ & $\begin{array}{l}\text { - IT during acupuncture treatment } \\
\text { - Carbon, EST, Ultra Sound, Hot Pack, } \\
\text { manual therapy, } 1 \text { time/day } \\
\text { - facial massage, spontaneous exercise } \\
\text { of expression muscles }\end{array}$ \\
\hline
\end{tabular}




\begin{tabular}{|c|c|c|c|c|}
\hline classification & subjects(onset) & tool & Korean medicine treatments & other treatment \\
\hline Ahn (2007) & $\begin{array}{l}\mathrm{M} / 54 \mathrm{Lt} \text {. facial palsy (2006.3) } \\
\mathrm{Hx} \text { after temporal bone fracture }\end{array}$ & $\begin{array}{l}\text { H-B scale } \\
\text { Y-system } \\
\text { DITI } \\
\text { EMG(electromyography) }\end{array}$ & $\begin{array}{l}\text { - } 0.30 \times 30 \mathrm{~mm} 2-3 \text { times/week, } 1 \text { time/week after } 2 \text { months, } 20 \text { mins } \\
\text { - electro-acupuncture: } 60 \mathrm{~Hz}, 5 \mathrm{~mA}, 20 \mathrm{mins} \\
\text { - herbal medicine } \\
\text { - } 1 \text { month starting from March } 29 \text { GamiLigigeopoong-san } \\
\text { - Starting from May Bogigeopung-san }\end{array}$ & $\begin{array}{l}\text { - low frequency therapy after acupuncture } \\
\text { treatment on affected-side of facial area (GD- } \\
751 \text {, Japan) } 5 \sim 6 \mathrm{~Hz}, 10 \text { mins } \\
\text { - combined with Laser therapy (DIOSS-620) } \\
\text { - facial massage, facial hot pack guidance at } \\
\text { home }\end{array}$ \\
\hline Cho (2016) & $\begin{array}{l}\text { F/72 Rt. Facial palsy (2016.4) } \\
\text { '96 Cesarean surgery } \\
\text { '00 Rheumatoid arthritis Dx. po-med } \\
(+) \\
\text { '05 HNP of L-spine(L4-5) Dx. }\end{array}$ & $\begin{array}{l}\text { H-B scale } \\
\text { Y-system } \\
\text { SFGS }\end{array}$ & $\begin{array}{l}\text { - } 0.30 \times 60 \mathrm{~mm} 2 \text { times/day, } 30 \mathrm{mins} \\
\text { - acupuncture points: BL2, GB14, TE23, ST4 etc } \\
\text { - electro-acupucnture: Whata } 153,1.5-2.0 \mathrm{G} \text { stimulation } \\
\text { - BL2 GB14 TE23 ST4 } \\
\text { - } 2 \text { chub } 3 \text { pack }-3 \text { times/day }(120 \mathrm{ml} / \text { pack }) \\
\text { - early stage of hospitalization GamiLigigeopoong-san } \\
\text { - after pain reduced and symptoms improved BojunglkgitangGagam }\end{array}$ & $\begin{array}{l}\text {-acupuncture-electrical stimulation } \\
\text { interference wave } \\
\text { - } 500 \mathrm{~Hz}, \mathrm{BL} 2-\mathrm{TE} 23 \mathrm{ST} 4-\mathrm{ST} 6 \\
\text { - early stage of onset- combined treatment of } \\
\text { antivirus agents and corticosteroids for } \\
\text { blisters and pain in auricular area (6 days) } \\
\text { - Prednisolone 5mg } \\
\text { - 3T->3T->3T->1T->1T->1T\#1 (B/pc 30min) }\end{array}$ \\
\hline Jang (2012) & F/45 Lt. Facial palsy (2010.8) & $\begin{array}{l}\text { Y-system } \\
\text { Subjective degree of } \\
\text { satisfaction evaluation } \\
\text { (level of symptom, } \\
\text { quality of treatment) }\end{array}$ & $\begin{array}{l}\text { - filiform acupuncture } 2 \text { times/week } \\
\text { - acupuncture points: LI4, Samjung, Cheuksamli, Cheukhasamli } \\
\text { (Dong`s Acupuncture) }\end{array}$ & $\begin{array}{l}\text { - thread embedding acupuncture } 1 \text { time } / 3 \\
\text { weeks, total } 2 \text { times } \\
\text { - sterilized } 4 \mathrm{~cm} \text { or } 6 \mathrm{~cm} \text { PDS inserted } 29 \mathrm{G} \\
\text { needle } \\
\text { - injected on SMAS of skin } \\
\text { - } 2 \text { points - procedure direction from jawbone } \\
\text { to angulus mandibulae, and upper side of } \\
\text { branches on the Lt. facial area } \\
\text { - } 4 \text { points - procedure direction from end of lip } \\
\text { to levator labii superioris alaeque nasi, } \\
\text { levator labii superioris, zugomaticus minor, } \\
\text { zygomaticus major following the muscle } \\
\text { grain - total } 6 \text { points }\end{array}$ \\
\hline Choi (2012) & $\begin{array}{l}\text { F/47 Rt. Facial palsy (2010.7) } \\
95 \text { gastrospasm treatment }- \text { improved }\end{array}$ & $\begin{array}{l}\text { Y-system } \\
\text { VAS(pain in ear) }\end{array}$ & $\begin{array}{l}-0.20 \times 30 \mathrm{~mm} 2 \text { times/day } \\
-1^{\text {st }} \text { treatment electro-acupuncture } 2-3 \mathrm{hz}, 20 \mathrm{mins} \\
-2^{\text {nd }} \text { treatment } 15 \mathrm{mins} \\
\text { - acupuncture points: EX-HN5, ST1, L120, ST4 etc }\end{array}$ & $\begin{array}{l}\text { - medication } \\
\text { - Ursa Tab 1T TidPC } \\
\text { - Reobil Tab 25mg 1T TidPC }\end{array}$ \\
\hline
\end{tabular}

\#1 M/50 Rt. Facial palsy (2004.11)

'96,98 pneumothorax surgery (2

times)

\#2 M/36 Rt. Rt. Facial palsy

(2005.1)

04 DM cognition -1 month of

medication

04.12 Tonsillitis Adm Tx.

\#3 M/37 Rt. Facial palsy (2005.3)

Lee (2006) 92 DM cognition med Qd po

- $0.30 \times 40 \mathrm{~mm} 1$ times/day

- affected-side: ST1 LI20 ST7 GV26 etc

- both sides: LI4 ST36

\#4 M/48 Lt. Facial palsy (2005.10)

- Perpendicular, Young-Su-Bo-Sa, ST4-ST6 Tu-ja in turn

- herbal medicine: GamiLigigyeonjeong-san, Ligigeopoong-san,

\#5 F/15 Rt. Facial palsy (2005.11)

BogigeopungsanGagam

- To stimulate recovery of the muscle strength of paralyzed side Kinesio tape attached to facial muscle and SCM following muscle directions, leave it on for 10 hours/day and proceed facial massage \#6 M/26 Rt. Facial palsy (2006.1) \#7 M/13 Rt. Facial palsy (2006.1) \#8 M/40 Lt. Facial palsy (2006.4) \#9 F/45 Lt. Facial palsy (2006.2) $\# 10 \mathrm{M} / 43$ Lt. Facial palsy (2006.4) \#11 M/49 Lt. Facial palsy (2006.6) \#12 M/45 Rt. Facial palsy (2006.6)

- Warming meridian therapy: Facial Hot pack, IR

- Circulating meridian therapy: SSP (facial area)

- $0.25 \times 40 \mathrm{~mm} 2$ times/day

- Lt. side: ST4 ST6 EX-HN4 BL2 etc

Kim (2016) F/21 Lt. Facial palsy (2015.3) Y-system

- Rt. side: ST36 SP6 LR3 LI4 etc

- both sides GB20
F/64 Lt. Facial palsy, Rt. dextroversion (abduction) (2002.8)

Kim (2003) Hx Cerebral infarction (Rt.), Y-system Hypertension and cardiovascular disease ( 7 years ago $)$
- herbal medicine: Jodeungsan

-2 chub 3 packs - $120 \mathrm{cc}, 3$ times/day

- $0.25 \times 40 \mathrm{~mm} 1$ time/day, 15-20 mins

- acupuncture points: GB14, BL2, BL1, ST2

- herbal medicine: Yangkyuksanwha-tang, Ligigeopoong-san,

Gyeonjeong-san, GamiBoik-tang
- IR during acupuncture treatment

- Rt. side LR3, GB39, SP6, TE5 small mugwort moxibustion (sticker moxibustion) 3 times/day

- psychotonic, gastrointestinal drugs, ulcer medication, anti-nausea medication

- warm moxibustion or bamboo salt moxibustion on affected side of facial area, 1-2 pieces/day

- Shingigu on CV12 CV4 2 pieces/day

- Circulating meridian therapy: affected side

of facial area by radiculopathy

- Straightening meridian therapy: Carbon

point ray therapy using beaming

- 1 time/day, 20 mins 


\begin{tabular}{|c|c|c|c|c|}
\hline classification & subjects(onset) & tool & Korean medicine treatments & other treatment \\
\hline Lee (2014) & $\begin{array}{l}\text { \#1 F/70 Rt. Facial palsy (2014.1) } \\
\text { ' } 86.2 \text { medication after diagnosed of } \\
\text { hepatitis b and hepatocirrhosis } \\
\text { ' } 13 \text { osteoporosis, tooth-fracture } \\
\text { '03 medication after diagnosed of } \\
\text { herpes zoster - improved } \\
\text { '14 cerebral aneurysm, facial palsy. } \\
\text { ER B-MRI } \\
\text { \#2 M/60 Lt. Facial palsy (2013.4) } \\
\text { '12 medication after diagnosed } \\
\text { Hypertension/D.M/Hyperlipidemia } \\
\text { '00 medication after diagnosed of } \\
\text { fatty liver }\end{array}$ & $\begin{array}{l}\text { H-B scale } \\
\text { Y-system } \\
\text { NRS (pain in and behind } \\
\text { the ear, ear pain) } \\
\text { Subjective measure } \\
\text { (improvement of } \\
\text { symptoms) }\end{array}$ & $\begin{array}{l}-0.25 \times 30 \mathrm{~mm} 1 \text { time/day, } 20 \mathrm{mins} \\
\text { - affected-side: BL2 EX-HN4 TE23 GB14 } \\
\text {-electro-acupuncture: continuous wave } 35 \mathrm{~Hz} \text {, identical acupuncture } \\
\text { points used } \\
\text { - original formula } 3 \mathrm{chub} / \text { day, } 3 \text { times } \\
\text { - \#1, \#2 Hyeongbangdojok-san }\end{array}$ & $\begin{array}{l}\text { - facial massage, physical therapy (EST) } \\
\text { - wet cupping on TE17 for sever pain in and } \\
\text { behind the ear }\end{array}$ \\
\hline Jung (2013) & $\begin{array}{l}\text { \#1 F/78 Rt. Facial palsy (2012.4) } \\
10 \text { medication after diagnosed of } \\
\text { cerebral infarction } \\
\text { 00 medication after diagnosed of } \\
\text { prostate cancer } \\
\text { '02 medication after diagnosed of gout } \\
\# 2 \mathrm{M} / 10 \text { Lt. facial palsy (2012.5) } \\
\text { 03 Occasional treatment of Korean } \\
\text { Medicine for atopic dermatitis } \\
\text { 06 hospitalized treatment for asthma }\end{array}$ & $\begin{array}{l}\text { H-B scale } \\
\text { Y-system } \\
\text { NRS (pain in and behind } \\
\text { the ear, ear pain) } \\
\text { Subjective measure } \\
\text { (improvement of } \\
\text { symptoms) }\end{array}$ & $\begin{array}{l}-0.25 \times 30 \mathrm{~mm} 1 \text { time/day, } 20 \mathrm{mins} \\
\text { - affected-side: GV26 CV24 EX-HN3 BL2 etc, Tonifications of Kidney } \\
\text { - electro-acupuncture: continuous wave } 35 \mathrm{~Hz} \text {, identical acupuncture } \\
\text { points } \\
\text { - original formula } 3 \mathrm{chub} / \text { day, } 3 \text { times } \\
\text { - \#1, \#2 Hyeongbangdojok-san }\end{array}$ & $\begin{array}{l}\text { - wet cupping } \\
\text { - \#1 first } 7 \text { days on TE17 } 1 \text { time/day } \\
\text {-\#2 no wet cupping considering the patient age } \\
\text { (child) } \\
\text { - } 3 \text { times/day facial massage on affected-side } \\
\text { of facial are and instructions for facial exercise }\end{array}$ \\
\hline Kim (2005) & $\begin{array}{l}\text { F/51 Lt. facial palsy (2004.10) } \\
\text { Hx cystitis }\end{array}$ & & $\begin{array}{l}\text { - acupuncture } \\
\text { - affected-side: ST4-ST6 tu-ja, GV26 CV24 ST2 GB14 LI4 ST36 GV20 } \\
\text { - herbal medicine: Ligigeopoong-tang }\end{array}$ & \\
\hline Kang (2010) & $\begin{array}{l}\text { F/41 Rt. facial palsy (2009.9) } \\
\text { Hx } 7 \text { month pregnancy }\end{array}$ & $\begin{array}{l}\text { Y-system } \\
\text { VAS(movement of facial } \\
\text { muscles, closing eyes, } \\
\text { chewing) }\end{array}$ & $\begin{array}{l}\text { - } 0.25 \times 40 \mathrm{~mm} 3 \text { times/week, } 20 \text { mins } \\
\text { - non-affected side: ST36 ST41 LI4 LR3 } \\
\text { - affected side: EX-HN4 BL2 LI20 Sanso (LI20 external lower part) etc } \\
\text { - electro-acupuncture } \\
\text { - 3Hz, } 20 \text { mins } \\
\text { - affected side: EX-HN4-BL2, LI20-Sanso, ST4-ST6 } \\
\text { - pharmacopuncture } \\
\text { - bee venom acupuncture (bee venom diluted in bamboo salt solution } \\
\text { by 10000:1) } \\
\text { - affected side: EX-HN4 SI18 ST4 LI20 0.01cc }\end{array}$ & \\
\hline Park (2002) & $\begin{array}{l}\text { F/43 facial palsy both sides }(2002.6) \\
\text { Hx Rt. Facial nerve paralysis } \\
\text { (Guanwasa) (age 16) - recovered } \\
\text { after Korean Medicine treatment }\end{array}$ & $\begin{array}{l}\text { H-B scale } \\
\text { DEFS (Detailed } \\
\text { Evaluation of } \\
\text { Facial } \\
\text { Symmetry) }\end{array}$ & $\begin{array}{l}\text { - acupuncture } \\
\text { - both sides: GB20 ST8 GB14 TE23 BL2 BL1 GB1 ST6 ST4 ST7 ST3 } \\
\text { LI20 LI19 GV26 CV24 LI4 } \\
\text { - electro-acupuncture } \\
\text { - both sides: ST7 ST6 } \\
\text { - herbal medicine: GamiLigigeopoong-san }\end{array}$ & $\begin{array}{l}\text { - medication: Methylon } 4 \mathrm{mg} 12 \mathrm{~T} \# 110 \text { days } \\
\text { after hospitalization } \\
\text { - IR on facial area, low frequency treatment, } \\
\text { visible ray synthesis therapy } \\
\text { - dry cupping on behind of ear }\end{array}$ \\
\hline Lee (2006) & $\begin{array}{l}\text { \#1 M/34 Lt. facial palsy ( } 2005.7 \text { ) } \\
05 \text { cerebral hemorrhage, Lt. temporal } \\
\text { bone fracture - cerebral hemorrhage } \\
\text { operation, decompression of facial } \\
\text { nerve } \\
\text { \#2 F/2 Lt. facial palsy ( } 2005.3 \text { ) } \\
05 \mathrm{Lt} \text {. external ear fracture and facial } \\
\text { paralysis by TA }\end{array}$ & Y-system & $\begin{array}{l}\# 1 \\
\text { - } 0.25 \times 30 \mathrm{~mm} 2 \text { times/day, } 25 \text { mins, Young-Su-Bo-Sa } \\
\text { - affected side BL2 GB14 TE23 GB1 etc } \\
\text { - non-affected side: SI6 LR3 LR2 GB41 etc } \\
\text { - electro-acupuncture: } 1 \text { times, } 15 \text { mins, BL2 GB14 LI20 SI18 etc } \\
\text { - herbal medicine: GamiBojunglkgi-tang } \\
\text { - Saenggangeonbi-tang } \\
\# 2 \\
\text { - } 0.25 \times 30 \mathrm{~mm} 2 \text { times/day, } 25 \mathrm{mins} \\
\text { - affected side: BL2 GB14 TE23 GB1 etc } \\
\text { - both sides: LI4 LR3 ST36 } \\
\text { - electro-acupuncture: } 1 \text { times, } 15 \text { mins, BL2 GB14 LI20 SI18 etc } \\
\text { - herbal medicine: Kamiboa-tang }\end{array}$ & $\begin{array}{l}\text { - medication } \\
\# 1 \text { Nicetile Tab, Ganaton Tab 50mg, } \\
\text { Phenytoin Tab 100mg, Selbex Cap, Sermion } \\
\text { Tab 10mg, Ursa Tab 3T\#3 } \\
\text { - IR during acupuncture treatment } \\
\text { - } 1 \text { time/day facial SSP on affected side } \\
\text { - head and neck area hot pack, negative } \\
\text { - facial massage on affected side of facial area } \\
\text { several times/day, } 10 \text { mins }\end{array}$ \\
\hline
\end{tabular}




\begin{tabular}{|c|c|c|c|c|}
\hline classification & subjects(onset) & tool & Korean medicine treatments & other treatment \\
\hline Choi (2002) & $\begin{array}{l}\text { M/36 Rt. facial palsy (2001.11) } \\
\text { '01 traumatic temporal bone fracture- } \\
\text { treatment process ongoing, facial } \\
\text { nerve paralysis }\end{array}$ & $\begin{array}{l}\text { H-B grade } \\
\text { Y-system } \\
\text { DITI }\end{array}$ & $\begin{array}{l}\text { - } 0.30 \times 30 \mathrm{~mm} 1 \text { time/day, } 20 \mathrm{mins} \\
\text { - affected side: GB20 ST8 ST6 ST4 etc } \\
\text { - non-affected side: ST36 } \\
\text { - both sides: LI4 } \\
\text { - pharmacopuncture: Sodam - Hominis Placenta - Junggi-haham } \\
\text { - herbal medicine: Ligigeopoong-san - Gamiboik-tang } \\
\text { (movement of head area starting) }\end{array}$ & $\begin{array}{l}\text { - IR on affected side of facial area, TDP, SSP } \\
\text { - facial massage } \\
\text { - wet cupping when severe pain in and behind } \\
\text { the ear }\end{array}$ \\
\hline Kang (2009) & F/53 Lt. facial palsy (2007.10) & $\begin{array}{l}\text { H-B grade } \\
\text { Y-system } \\
\text { VAS(facial and parotoid are } \\
\text { pain) }\end{array}$ & $\begin{array}{l}\text { - } 0.35 \times 40 \mathrm{~mm} 2 \text { times/day, } 25 \mathrm{mins} \\
\text { - affected side: ST6 ST4 BL2 TE17 etc } \\
\text { - blood-letting when severe pain in and behind the ear on TE17 } \\
\text { - herbal medicine: GamiLigigeopoong-san, } 3 \text { times } / \text { day, } 120 \mathrm{ml}\end{array}$ & \\
\hline Shin (2008) & $\begin{array}{l}\text { \#1 F/28 Rt. facial palsy }(2008.1) \\
\text { '97 Allergic rhinitis } \\
\text { '06 Laser surgery } \\
\text { \#2 F/31 Lt. facial palsy }(2007.5)\end{array}$ & Y-system & $\begin{array}{l}\text { - } 0.25 \times 30 \mathrm{~mm} 2 \text { times/day (inpatient) } 1 \text { time/day (outpatient), } 20 \text { mins } \\
\text { - acupuncture points: ST2 TE23 GB14 ST4 etc } \\
\text { - electro-acupuncture: BL2 GB14 LI20 SI18 etc } \\
\text { - herbal medicine } \\
\text { - \#1 GamiDangkwi-San } 20 \text { chub } \\
\text { - \#2 Sanhu-A-Gamibang } 30 \text { chub }\end{array}$ & $\begin{array}{l}\text { - IR during acupuncture treatment } \\
\text { - facial SSP, manual therapy on facial area, } 1 \\
\text { time/day } \\
\text { - facial area exercise, several times/day }\end{array}$ \\
\hline Lee (2005) & F/30 Lt. facial palsy (2004.1) & $\begin{array}{l}\text { H-B grade } \\
\text { Paralysis level of facial } \\
\text { muscle }(-\sim++++)\end{array}$ & $\begin{array}{l}\text { - } 0.30 \times 40 \mathrm{~mm} 1 \text { time/day, } 15-20 \mathrm{mins} \\
\text { - acupuncture points: ST2 TE23 GB14 ST4 etc } \\
\text { - nausea during pregnancy treating points: PC6 } \\
\text { - herbal medicine: AntaesanGami-GamiBosaeogtang-- } \\
\text { AntaeeumGami-PaljintangGami }\end{array}$ & $\begin{array}{l}\text { - facial massage, } 2 \text { times/day, } 10 \text { mins } \\
\text { - PTx: IR on affected side of facial area during } \\
\text { acupuncture treatment } \\
\text { - laser therapy on identical acupuncture points } \\
\text { by every } 2 \text { mins }\end{array}$ \\
\hline Choi (2003) & $\begin{array}{l}\text { F/24 Lt. facial palsy (2003.3) } \\
\text { ' } 99 \text { gastritis, gastric ulcer } \\
\text { '01 artificial abortion } \\
\text { '02 spontaneous abortion }\end{array}$ & $\begin{array}{l}\text { DITI } \\
\text { Observation of symptom } \\
\text { Change during } \\
\text { hospitalization period } \\
\text { Grading system of facial } \\
\text { palsy }\end{array}$ & $\begin{array}{l}\text { - } 0.30 \times 30 \mathrm{~mm} 1 \text { time/day, } 15 \text { mins } \\
\text { - affected side: ST6 ST4 BL2 TE23 LI20 CV24 ST2 GB14 } \\
\text { - non-affected side: LI4 } \\
\text { - herbal medicine: Pyungweesan } 3 \text { times/day x 7days }\end{array}$ & - facial massage \\
\hline $\operatorname{Kim}(2005)$ & $\begin{array}{l}\text { M/54 Lt. facial palsy (2004.9) } \\
\text { M/44 Lt. facial palsy (2004.9) } \\
\text { M/36 Lt. facial palsy (2004.11) } \\
\text { M/71 Lt. facial palsy (2004.10) } \\
\text { 02 TA, subdural hemorrhage op. } \\
\text { F/35 Rt. facial palsy (2004.11) } \\
\text { Hx Age } 15,25 \text { Rt. facial palsy } \\
\text { F/49 Rt. facial palsy (2004.11) } \\
\text { Hx DM, Hyperlipidemia } \\
\text { F/47 Rt. facial palsy (2004.11) } \\
\text { Hx Age 22 Lt. facial palsy }\end{array}$ & H-B scale & $\begin{array}{l}\text { - } 0.30 \times 30 \mathrm{~mm} 1 \text { time/day Young-Su-Bo-Sa, ST4-ST6 Tu-ja in turn } \\
\text { - affected side: ST1 ST4 ST6 ST7 etc } \\
\text { - both sides: LI4 ST36 } \\
\text { - non-affected side: Sam-hwang Points } \\
\text { - herbal medicine: GamiLigigyeonjeong-san, Ligigeopoong-san, } \\
\text { BogigeopungsanGagam }\end{array}$ & $\begin{array}{l}\text { - Prednisone } 1 \mathrm{~kg} / \mathrm{lmg} \text { and reduced during } 10 \\
\text { days } \\
\text { - Taping therapy - attached on facial muscles } \\
\text { following muscle directions } \\
\text { - facial massage(with taping on) } \bullet \text { Facial Hot } \\
\text { pack, IR, SSP on facial area }\end{array}$ \\
\hline $\operatorname{Kim}(2006)$ & $\begin{array}{l}\text { F/34 Rt. facial palsy (2001.7) } \\
99 \quad \text { Rt. Facial numbness after } \\
\text { microvascular decompression } \mathrm{F} / 32 \\
\text { Rt. facial palsy (2004.4) } \\
\text { '04 microvascular decompression } \\
\text { F/35 Rt. facial palsy (2002.4) } \\
02 \text { brain surgery }\end{array}$ & $\begin{array}{l}\text { H-B grade } \\
\text { Y-system } \\
\text { electroneurography } \\
\text { (evaluation of rate of } \\
\text { neurodegeneration) } \\
\text { Sequela symptom evaluation }\end{array}$ & $\begin{array}{l}\text { - } 0.25 \times 40 \mathrm{~mm} \text { deviation of treatment number depending on patient } \\
\text { - non-affected side: ST6 ST4 BL2 TE17 etc } \\
\text { - both sides: LI11 ST36 } \\
\text { - herbal medicine: Gwakhyangjunggi-san gamibang } \\
\text { depending on patient condition Gamiboik-tang, GamiSeunggaltang }\end{array}$ & $\begin{array}{l}\text { - After } 20 \text { mins warm-up, TDP on affected } \\
\text { side of facial area during acupuncture } \\
\text { treatment from } 30 \mathrm{~cm} \text { distance } \\
\text { - aroma therapy }\end{array}$ \\
\hline Lee (2009) & $\begin{array}{l}\text { F/64 Rt. facial palsy (2009.3) } \\
98 \text { D.M cognition - on medication } \\
08 \text { Breast cancer - onset during } \\
\text { anticancer treatment after surgery } \\
\text { F/34 Lt. facial palsy (2008.11) } \\
94,04 \text { Lt. facial palsy } \\
\text { 08 Onset during anticancer treatment } \\
\text { after gastrotomy diagnosed of gastric } \\
\text { cancer } \\
\text { F/69 Rt. facial palsy (2009.1) } \\
08 \text { Breast cancer, onset during } \\
\text { anticancer treatment after surgery }\end{array}$ & Y-system & $\begin{array}{l}\text { - } 0.25 \times 30 \mathrm{~mm} 2 \text { times/day, } 20 \text { mins } \\
\text { - morning, affected side: TE23 BL2 ST2 GB14 etc } \\
\text { - afternoon, affected side: LI4, Samjung, Cheuksamli, } \\
\text { Cheukhasamli(Dong`s Acupuncture) etc } \\
\text { - electro-acupuncture: BL2 GB14 ST4 ST6 } \\
\text { - herbal medicine } \\
\text { - acute pain in and behind the ear: Wugakseongma-tang, } \\
\text { Bangpungtongsung-san - Pain reduced - Samchulkunbitang, } \\
\text { Samryungbaikchul-san depending on patient condition }\end{array}$ & $\begin{array}{l}\text { - IR on affected side of facial area during } \\
\text { acupuncture treatment } \\
\text { - SSP, facial massage, facial muscle exercise } \\
1 \text { time/day } \\
\text { - Head and neck area ICT, dry cupping } \\
\text { - medication } \\
\# 1 \text { anticancer medicine, calcium agent, } \\
\text { antihyperlipidemic agent, hypoglycemic } \\
\text { agent } 1 \mathrm{~T} \# 1 \\
\text { Oral hypoglycemic agent ( } 2 \text { types) } 2 \mathrm{~T} \# 2 \text {, } \\
1.5 \mathrm{~T} \# 1 \\
\# 2 \text { prokinetic drugs, anticonvulsant, digestive } \\
\text { medicine, anticancer agent, intestinal disorder } \\
\text { agent 3C\#3 } \\
\# 3 \text { prokinetic drugs, anticancer agent, ulcer } \\
\text { medicine } 1 \mathrm{~T} \# 1\end{array}$ \\
\hline
\end{tabular}

H-B scale: House-Brackmann Grading System; Y-system: Yanagihara grading system; DITI: Digital infrared thermal imaging; NRS: Numeral rating scale; VAS: Visual analog scale; ENoG: Elecrtonerography; EMG: electromyogram; SFGS: Sunnybrook facial grading system; IR: Infrared ray; SSP: Silver spike point; EST: Electrical Stimulation; FES: Functional Electronical Stimulation; ICT: Inter current therapy; MET: Myoneural Excitability Test; D.M: Dermatomyositis; Od: Oculus dexter; Fx: Fracture; HIVD: Herniated intervertebral disk; O.A.: osteoarthritis; W.N.L: within normal limits; HNP: herniated nucleus pulposus 
documented in <Donguibogam $>$ [23]. It also influences removing and scattering wind-cold and moderating qi and vitalizing meridians and used for early stage symptoms of Guanwasa [13]. Bojunglkgi-tang was frequently used for fundamental treatment of deficient-type Guanwasa after general symptoms improved [24]. Ligigeopoong-san was also used in some cases until symptoms were improved, and Gamiboik-tang was used when facial area movement occurred [25]. Gyeonjeong-san is a formula frequently used in Donguibogam, well-known for Guanwasa caused by stroke. Medication in conventional medicine was used to treat preexisting diseases, often combining steroids or antivirus agents with herbal medicine. However, most of the cases did not document the purpose of the medication, which caused difficulty in conducting research based on the reference.

Other treatments included IR, facial massage and SSP. When infrared light is exposed to the affected area it is shown to have calming effect and relieve pain, and is considered to be a main treatment of facial palsy [26]. SSP is a low frequency electric-stimulating treatment device using silver plate electrodes on the body surface. Studies show that when electro-acupuncture is used with SSP treatment the treatment rate increases which is why SSP treatment is used frequently [27].

This study has analyzed 18 years of studies on facial palsy treatment and has reviewed diagnosis, evaluating tools, and treatment tools related with facial palsy treatment. However, it has limits since an international database was not used within the study, and the searching words were limited to 'facial palsy' and 'Guanwasa' which does not cover all studies on facial palsy. This study shows case reports and the diagnosis, evaluating tools and treatment tools used for facial palsy and the frequency of use. Based on this study, additional research is needed on the use of common treatment tools to review how the treatment rate differs.

\section{Conclusion}

To review the domestic trend of facial palsy and the diagnosis, evaluating tools, and treatment tools, 37 case reports were analyzed

(1) Thirty-five studies (94.5\%) used evaluating tools to measure the patients' improvement rate. The H-B scale (23 times, 65.7\%) and Y-system (22 times, 62.8\%) were frequently used.

(2) All studies used filiform acupuncture needles, 19 studies (51.3\%) used additional electro-acupuncture, and 8 studies (21.6\%) used additional pharmacopuncture. Frequently used acupuncture points were ST6, ST4, GB14, TE23, LI20, BL2, and GV26.

(3) Thirty-two studies (86.4\%) used herbal medicine while 12 studies $(32.4 \%)$ used conventional medicine medication. Ligigeopoong-san, Bojunglkgi-tang, and Gyeonjeong-san were frequently used.

(4) Twenty-nine studies $(78.3 \%)$ used other treatments including IR, facial massage and SSP.
Facial palsy treatment uses a wide-range of therapeutic methods, mainly acupuncture with electro-acupuncture and pharmacopuncture, mostly combined with herbal medicine.

\section{Acknowledgement}

This research achievement is conducted with assistance from Ministry of Health and Welfare through the Korea Health Industry Development Institute (HB16C0028).

\section{Conflict of interest}

The authors declare that there are no conflicts of interest.

\section{References}

1. Kim DH, Kim JS, Choi JH, et al. The Review of Electrophysiological Test and Therapeutic Approach in Facial Nerve Injury. 2000;39(2):61-62.

2. Korean Acupuncture \& Moxibustion Medicine Society. Theacupuncture and moxibustion medicine 2nd vol. Gyeonggi:Jipmoondang. 2008;186-90.

3. Kim MS, Lee EY. A case report of the Bell`s palsy patient which accompanies trigeminal neuralgia. The journal of clinical thesis korean acupuncture \& Moxibustion Society. 2003;0(0):46-52.

4. Kim MB, Kim JH, Sin SH, Yoon HJ, Kho WS. A study of facial nerve grading system. The Society of Korean Medicine Ophthalmology, Otolaryngology \& Dermatology. 2007;20(3):147-160.

5. Fauci, Braunwald, Kasper, et al. Harrison's Principles of Internal Medicine. 13th ed. Seoul:Jeongdam; 1997.

6. Lee SJ, Kim HJ, Seo DW, et al. Analysis of Clinical Outcome and Effectiveness of Steroid and Antiviral Treatment in Patients with Bell`s Palsy in the Emergency Department. Journal of the Korean Society of Emergency Medicine. 2012;23(4):531-536.

7. Sim SY. Clinical Research of Korean Medical Treatment for the Peripheral Facial Paralysis. Journal of Korean Medicine Ophthalmology \& Otolaryngology \& Dermatology. 2015;28(4):62-73.

8. Lee EJ, Kim ST, Kwon MG, et al. Journal of Physiology \& Pathology in Korean Medicine. 2015;29(4):347-351.

9. Chu MG, Jo HG, Choi JB, et al. Clinical case study of facial nerve palsy using facial acupuncture. Journal of Physiology \& Pathology in Korean Medicine. 2009;23(5):1188-1192.

10. Jang YJ, Yang TJ, Shin JC, et al. Clinical Case Study of Facial Nerve Paralysis with Sensorineural Hearing Loss and Tinnitus Caused by Traumatic Temporal Bone Fracture. THE ACUPUNCUTURE. 2016;33(1):95-101.

11. Lee JM, Kim EM, Song HG, et al. Clinical study of two patients with deveation of the eye and mouth caused by trauma. The journal of Korean Acupuncture \& Moxibustion Society. 2006;23(4):81-89.

12. Kim JI, Seo JC, Lee SH, Choi DY, Kang SK, Koh HK. The clinical observation on Bell's palsy according to facial nerve grading system. The journal of Korean Acupuncture \& Moxibustion Society. 2002;19(5):112-123. 
13. Kim JI, Kho HG, Kim CH. A study of facial nerve grading system. The journal of Korean Acupuncture \& Moxibustion Society. 2001;18(2):1-17.

14. Hwang CY, Lee BL, Hwang JY. A Literature Review on the Acupoint of Bell's Palsy. Research Institute of Korean Medicone. 1998;7(1):817-829.

15. Hong KJ, An SH, Kim JH, Hwang JH, Kim KS, Sohn IC. Effects of Acupuncture at Hap - Kok ( LI4 ) on the Skin Temperature Changes of face divided by 17 area randomly in Man. The journal of Korean Acupuncture \& Moxibustion Society. 2002;19(1):24-38.

16. An BJ, Song HS. Effect of Electroacupuncture on Patients with Peripheral Facial Paralysis. The journal of Korean Acupuncture \& Moxibustion Society. 2005;22(4):121-129.

17. Commission of Publishing textbook of Acupuncture. Acupuncture. Jipmoondang. 2008;364-70:483-502.

18. Lee JH. Electrotherapy. Seoul: Daihakseorim. 1995;5377:273-304

19. Sin MS, Park CJ, Choi SW, Yook TH. Clinical Studies on Herbal Acupuncture Therapy in Peripheral Facial Palsy. Journal of Yakchim (Korean of Herb-Acupuncture) Institute. 2001;4(2):27-33.

20. Kwon KR. The study of the introduction of Bee venom Acupuncture, Biochemistry and Pharmachology have been obtained the following results, The journal of Korean Acupuncture \& Moxibustion Society. 1994;11(1):159-171.

21. Cho JY, Kim YJ, Kim EJ, Lee SD, Kim KS. The Effects of Hwangryunhaedok-tang Pharmacopuncture by the Anti-inflammatory Action of Suppression of iNOS Production on Mice with Allergic Rhinitis. The journal of Korean Acupuncture \& Moxibustion Society. 2012;29(1):89-101.

22. Park CW, Ahn Ks, Choi SJ, Munh DS. A Clinical Study of Facial Nerve Paralysis. Korean J Otolaryngol. 1998;41(4):430-435.

23. Heo J. Donguibogam. Seoul:Yeogang Publishing Company. 2001;1385,1387,2307.

24. Kim BS, Lim HY, Kim SM, Park JH, LEE TH. A Clinical Report on One Case of Nuclear Facial Nerve Paralysis. The Journal of Korean Oriental Internal Medicine. 2003;24(2):395-401.

25. Choi SW. Clinical Study of Patient with Facial Nerve Paralysis Caused by Traumatic Temporal Bone Fracture. The journal of Korean Acupuncture \& Moxibustion Society. 2002;19(3):207-215.

26. The Korean Academy of Oriental Rehabilitation Medicine. Oriental Rehabilitation Medicine. 2nd ed. Seoul: Koonja Publishing Company. 2005;317-8,336,338.

27. Hwang JH, Lee DG, LEE HJ, Cho HS, Kim KH, Kim KS. Effect of Combined Silver Spike Point Therapy and Electroacupuncture on Patients with Peripheral Facial Paralysis. The journal of Korean Acupuncture \& Moxibustion Society. 2007;24(4):69-80. 